\title{
National Endoscopy Quality Improvement Program Remains Suboptimal in Korea
}

\author{
Jae Myung Cha ${ }^{1}$, Jeong Seop Moon², Il-Kwun Chung ${ }^{3}$, Jin-Oh Kim³ ${ }^{3}$, Jong Pil Im ${ }^{4}$, Yu Kyung Cho ${ }^{5}$, Hyun Gun Kim ${ }^{3}$, Sang Kil \\ Lee $^{6}$, Hang Lak Lee ${ }^{7}$, Jae Young Jang ${ }^{1}$, Eun Sun Kim ${ }^{8}$, Yunho Jung ${ }^{3}$, Chang Mo Moon ${ }^{9}$, Yeol Kim ${ }^{10}$, and Bo Young Park ${ }^{10}$ \\ ${ }^{\prime}$ Department of Internal Medicine, Kyung Hee University School of Medicine, ${ }^{2}$ Department of Internal Medicine, Inje University College of \\ Medicine, ${ }^{3}$ Department of Internal Medicine, Soonchunhyang University College of Medicine, ${ }^{4}$ Department of Internal Medicine, Seoul National \\ University Hospital, ${ }^{5}$ Department of Internal Medicine, The Catholic University of Korea College of Medicine, ${ }^{6}$ Department of Internal Medicine, \\ Yonsei University College of Medicine, ${ }^{7}$ Department of Internal Medicine, Hanyang University School of Medicine, ${ }^{8}$ Department of Internal \\ Medicine, Korea University College of Medicine, ${ }^{9}$ Department of Internal Medicine, Ewha Womans University School of Medicine, Seoul, and \\ ${ }^{10}$ Cancer Early Detection Branch, National Cancer Control Institute, National Cancer Center, Goyang, Korea
}

\section{See editorial on page 657.}

Background/Aims: We evaluated the characteristics of the National Cancer Screening Program (NCSP) and opinions regarding the National Endoscopy Quality Improvement Program (NEQIP). Methods: We surveyed physicians performing esophagogastroduodenoscopy and/or colonoscopy screenings as part of the NCSP via e-mail between July and August in 2015. The 32-item survey instrument included endoscopic capacity, sedation, and reprocessing of endoscopes as well as opinions regarding the NEQIP. Results: A total of 507 respondents were analyzed after the exclusion of 40 incomplete answers. Under the current capacity of the NCSP, the typical waiting time for screening endoscopy was less than 4 weeks in more than $90 \%$ of endoscopy units. Performance of endoscopy reprocessing was suboptimal, with $28 \%$ of respondents using unapproved disinfectants or not knowing the main ingredient of their disinfectants and $15 \%$ to $17 \%$ of respondents not following reprocessing protocols. Agreement with the NEQIP was optimal, because only $5.7 \%$ of respondents did not agree with NEQIP; however, familiarity with the NEQIP was suboptimal, because only $37.3 \%$ of respondents were familiar with the NEQIP criteria. Conclusions: The NEQIP remains suboptimal in Korea. Given the suboptimal performance of endoscopy reprocessing and low familiarity with the NEQIP, improved quality in endoscopy reprocessing and better understanding of the NEQIP should be emphasized in Korea. (Gut Liver 2016;10:699-705)
Key Words: Endoscopy, gastrointestinal; Quality; Stomach neoplasms; Colorectal neoplasms; Mass screening

\section{INTRODUCTION}

Gastric cancers and colorectal cancers (CRCs) are the leading cause of cancer-related death in Korea. In Korea, nationwide screening for gastric cancer and CRC have been performed as a part of National Cancer Screening Program (NCSP) for Medical Aid recipients and National Health Insurance beneficiaries in the lower 50\% income bracket. ${ }^{1}$ For gastric cancer screening, either esophagogastroduodenoscopy (EGD) or gastrointestinal series are provided biennially for adults aged 40 years or older. ${ }^{2}$ For CRC screening, fecal occult blood tests (FOBTs) are provided annually as the primary screening method for adults aged 50 years or older, and follow-up investigation by either colonoscopy or double contrast barium enema are provided for individuals with a positive FOBT.

To improve the endoscopic quality of NCSP, the Korean Society of Gastrointestinal Endoscopy (KSGE) developed the National Endoscopy Quality Improvement Program (NEQIP)..$^{5-8}$ This program incorporates qualifications of endoscopists, quality improvement for instruments available at the endoscopy unit and endoscopic procedures (including sedation and reprocessing of endoscopes), as well as measurement of outcomes of endoscopy screening. ${ }^{5-8}$ Recently, many quality indicators for gastrointestinal endoscopy were published, ${ }^{9-11}$ and the importance of quality improvement in gastrointestinal endoscopy has been underlined. Although a better understanding of the characteristics of

Correspondence to: Jeong Seop Moon

Department of Internal Medicine, Seoul Paik Hospital, Inje University College of Medicine, 1342 Dongil-ro, Nowon-gu, Seoul 01757, Korea

Tel: +82-2-2270-0012, Fax: +82-2-2279-4021, E-mail: moonjs22@naver.com

Received on December 6, 2015. Revised on January 22, 2016. Accepted on January 22, 2016. Published online June 13 , 2016

pISSN 1976-2283 eISSN 2005-1212 http://dx.doi.org/10.5009/gnl15623

@ This is an Open Access article distributed under the terms of the Creative Commons Attribution Non-Commercial License (http://creativecommons.org/licenses/by-nc/4.0) which permits unrestricted non-commercial use, distribution, and reproduction in any medium, provided the original work is properly cited. 
nationwide screening endoscopy, endoscopic capacity, current state of sedation and reprocessing of endoscope, and opinion for NEQIP may be the first step towards a successful widespread adoption of nationwide screening endoscopy, the current state of these data for screening endoscopies have never been evaluated in Korea.

In this context, we evaluated the current status of the NCSP, endoscopic capacity, sedation and reprocessing, as well as opinion for NEQIP in Korea.

\section{MATERIALS AND METHODS}

\section{Study subjects}

This survey was conducted between July 2015 and August 2015 for all physician performing screening EGD and/or colonoscopy as a part of the NCSP. Physicians who were performing screening EGD and/or colonoscopy of NCSP in 2014 and members of the KSGE were selected for potential respondents. Physicians were excluded from the study if a valid e-mail address could not be located or if the questionnaire was not answered completely. The KSGE Ethics and Quality Improvement Committee approved the questionnaire. This study was approved by the Institutional Review Board at the Kyung Hee University Hospital at Gangdong (KHNMC IRB 2015-07-015).

\section{Questionnaire development and survey administration}

A 32-item survey instrument was developed based on literature review ${ }^{5-11}$ and semistructured discussions with community and academic gastroenterologists. Members of the Ethics and Quality Improvement Committee of KSGE also reviewed the survey instrument. The survey was designed to determine the current state of the NCSP, the endoscopic capacity, sedation, reprocessing of endoscopes, as well as opinion for NEQIP. The questionnaire was piloted among gastroenterology fellows of the Kyung Hee University Hospital at Gangdong. Feedback from this group was used to establish face and content validity and to revise the content of the survey instrument. Reported completion times ranged from 15 to 20 minutes. A link to a web-based version of the survey using the host SurveyMonkey (http:// www.surveymonkey.com) was e-mailed to all potential respondents. All responses were anonymous and voluntary. In this survey system, duplicated submissions from the same respondent were prevented. We offered token incentives, including W100,000 gift card, for three participants by random sampling. We sent a reinvitation e-mail to all potential respondents for three times, and the web-based survey was closed after 4 weeks.

\section{Statistical analysis}

Descriptive statistics were used to summarize sociodemographic characteristics. Categorical data were expressed as number (percentage), whereas continuous data were expressed as mean \pm standard deviations. Statistical analyses were performed using the SPSS version 18.0 for Windows (SPSS Inc., Chicago, IL, USA).

\section{RESULTS}

\section{Characteristics of respondents and their endoscopy units}

A total of 547 physicians responded to the web-based survey, with a total response rate of 18.8\% (547/2,906). Overall, 507 survey results were analyzed after exclusion of 40 incomplete answers. Table 1 shows the characteristics of respondents and endoscopy units performing screening endoscopies in NCSP. The respondents included 393 men (77.5\%) and 114 women $(22.5 \%)$, with a mean age of $41.9 \pm 7.3$ years. Most respondents $(84.2 \%)$ were gastroenterologists with fellow training more than 1 year, sufficient cumulative endoscopy volume, and continuous medical education for endoscopy. For provider specialty, most endoscopies were performed by a gastroenterologist ( 63.3\%), followed by an internal physician (25.4\%), and by other specialties (8.2\%). Approximately $41.0 \%$ of respondents were working at primary clinics/hospitals, and the remainder in tertiary

Table 1. Characteristics of Survey Respondents and Respective Endoscopy Units Participating in the National Cancer Screening Program

\begin{tabular}{|c|c|}
\hline Characteristic & Value \\
\hline \multicolumn{2}{|l|}{ Survey respondents } \\
\hline Age, yr & $41.9 \pm 7.3$ \\
\hline Male sex & $393(77.5)$ \\
\hline \multicolumn{2}{|l|}{ Specialty* } \\
\hline Gastroenterologists & $342(67.5)$ \\
\hline Other specialties (including internal medicine) & $163(32.1)$ \\
\hline Fellowship for endoscopy training $\geq 1 \mathrm{yr}$ & $427(84.2)$ \\
\hline \multicolumn{2}{|l|}{ Cumulative endoscopy volume } \\
\hline EGD $\geq 500$ cases & $484(95.5)$ \\
\hline Colonoscopy $\geq 150$ cases & $469(92.5)$ \\
\hline Continuous medical education for endoscopy & $485(95.7)$ \\
\hline \multicolumn{2}{|l|}{ Endoscopy unit } \\
\hline \multicolumn{2}{|l|}{ Practice site } \\
\hline Primary clinic/hospital & $208(41.0)$ \\
\hline Secondary hospital & $94(18.5)$ \\
\hline Tertiary or training hospital & $174(34.3)$ \\
\hline Others (military hospital/health promotion center) & $31(6.1)$ \\
\hline \multicolumn{2}{|l|}{ Proportion of specialties in the endoscopy unit } \\
\hline Gastroenterologists & $63.3(60.3-66.4)$ \\
\hline Internal medicine (excluding gastroenterology) 2 & $25.4(22.6-28.2)$ \\
\hline Others & $8.2(6.6-9.8)$ \\
\hline No. of endoscopy examination rooms & $3.5 \pm 3.2$ \\
\hline $\begin{array}{l}\text { Data are presented as mean } \pm \text { SD, number }(\%) \text {, or perce } \\
\text { EGD, esophagogastroduodenoscopy; CI, confidence in } \\
\text { *Two general practitioners respondents were excluded } \\
\text { ysis. }\end{array}$ & $\begin{array}{l}\text { ent }(95 \% \mathrm{CI}) . \\
\text { nterval. } \\
\text { d from the anal- }\end{array}$ \\
\hline
\end{tabular}


or training hospitals (34.3\%), secondary hospitals (18.5\%), and other institutions (6.1\%). The mean number of endoscopy examination rooms was 3.5 per endoscopy units, and 31.6\% of endoscopy units consisted of only one endoscopy examination room.

\section{Endoscopic capacity of the NCSP}

Under current capacity of NCSP, typical waiting time for screening endoscopy was less than 4 weeks in more than 90\% (93.5\% for EGD and 90.7\% for colonoscopy). Respondents estimated the potential maximum weekly number of endoscopies in addition to those in current practice that could be performed with current available resources. The potential maximum weekly number of EGDs and colonoscopies was about 54 and
17 cases, respectively. Respondents were asked about the overall obstacles (multiple choices) and three major obstacles (single choice) to perform more screening endoscopies at their practice site to accommodate an increased endoscopic demand (Table 2). The major three limiting factors were insufficient financial reimbursement (50.1\%), insufficient time (12.2\%) and insufficient nursing staffs (9.3\%). In a separate survey question, the respondents were asked about the strategies that would be considered in practice to accommodate an increased endoscopic demand. The most common response was that physicians would not consider performing more endoscopies (38.9\%). Respondents were next more likely to increase the proportion of working hours allocated to endoscopic procedures (16.0\%) and would hire more nursing staffs to assist with endoscopies (11.8\%).

Table 2. Endoscopic Capacity in the National Cancer Screening Program

\begin{tabular}{|c|c|}
\hline Endoscopic capacity & Value \\
\hline \multicolumn{2}{|l|}{ Typical waiting time (<4 wk) for endoscopy } \\
\hline EGD & $474(93.5)$ \\
\hline Colonoscopy & $460(90.7)$ \\
\hline \multicolumn{2}{|l|}{ Potential maximum number of endoscopies in addition to current practice } \\
\hline No. of EGD per week & $53.6 \pm 96.8$ \\
\hline No. of colonoscopy per week & $17.0 \pm 31.0$ \\
\hline \multicolumn{2}{|l|}{ Limiting factors to performing more endoscopies (multiple choices) } \\
\hline Insufficient time & $265(52.3)$ \\
\hline Insufficient utilization due to cancellations or no shows & $208(41.0)$ \\
\hline Insufficient number of physicians & $168(33.1)$ \\
\hline Insufficient nursing staff & $284(56.0)$ \\
\hline Insufficient procedure rooms, preparatory and/or recovery areas & $263(51.9)$ \\
\hline Insufficient endoscopes or monitors & 199 (39.3) \\
\hline Insufficient reimbursement & $384(75.7)$ \\
\hline \multicolumn{2}{|l|}{ Major obstacles to performing more endoscopies (single choice) } \\
\hline Insufficient reimbursement & $254(50.1)$ \\
\hline Insufficient time & $63(12.2)$ \\
\hline Insufficient nursing or ancilary staff & $47(9.3)$ \\
\hline \multicolumn{2}{|c|}{ Strategies taken to meet the increased endoscopic demand (multiple choices) } \\
\hline Not applicable, not planning to perform more endoscopies & 275 (54.2) \\
\hline Increase working hours allocated to endoscopy & $254(50.1)$ \\
\hline Use of reminder calls to decrease cancellations or no shows & $272(53.6)$ \\
\hline Increase medical staff & $237(46.7)$ \\
\hline Increase nursing staff & $329(64.9)$ \\
\hline Establish more procedure rooms, prep and/or recovery areas & $269(53.1)$ \\
\hline Purchase or lease more equipment & $255(50.3)$ \\
\hline \multicolumn{2}{|l|}{ Major strategies to meet the increased endoscopic demand (single choice) } \\
\hline Not applicable, not planning to perform more endoscopies & 197 (38.9) \\
\hline Increase working hours allocated to endoscopy & $81(16.0)$ \\
\hline Increase nursing staff & $60(11.8)$ \\
\hline
\end{tabular}

Data are presented as number $(\%)$ or mean \pm SD.

EGD, esophagogastroduodenoscopy. 


\section{Sedation and reprocessing of endoscopy}

For the survey of sedation (Table 3), two respondents were excluded from the analysis since sedative endoscopy was not performed in their endoscopy units. The most commonly used sedative agents were a combination of benzodiazepine and propofol (58.2\%), followed by benzodiazepine alone (30.1\%), propofol alone (7.3\%), a combination of etomidate and other agents (3.4\%), or other agents (1.0\%). Majority of physicians obtained informed consent and monitored patients for conscious sedation (95.2\% and 96.2\%, respectively).

In the survey for reprocessing of endoscopes (Table 3), 103 respondents were excluded from the analysis due to incomplete answers. The most commonly used disinfectants were glutaraldehyde (27.5\%), followed by ortho-phthalaldehyde (24.5\%), peracetic acid (13.1\%), and superoxidized water (6.9\%). However, 28.0\% of respondents used disinfectants that had not been approved by the Korean Food and Drug Administration or didn't know main ingredient of their disinfectants. Approximately $15 \%$ to $17 \%$ of respondents did not follow reprocessing protocols, i.e., $17.3 \%$ of respondents did not sterilize accessories that break the mucosal barrier and 15.1\% did not perform high-level disinfection for accessories attached to the endoscope. Most (84.2\%) of respondents underwent continuous medical education for reprocessing of endoscopic equipment.

\section{Opinions for the NEQIP}

For survey items regarding opinions for NEQIP (Table 4), 137 respondents were excluded from the analysis because of incomplete answers. Only $37.3 \%$ of respondents were very familiar or familiar with NEQIP criteria. Common reasons for gaps between NEQIP criteria and clinical practice were that "physician could not follow NEQIP criteria in clinical practice" (43.5\%) or "physician was not familiar with NEQIP criteria" (32.4\%). Over 60\% of respondents agreed that "NEQIP provides benefits for many of their patients" and "NEQIP would improve early diagnosis of gastric cancer or CRC." However, 66.8\% and 58.1\% of respondents were concerned about "NEQIP could be used in physician discipline" and "NEQIP could decrease physician reimbursement," respectively. Nearly half of the respondents agreed on the necessity of revising the NEQIP criteria, and only $14.6 \%$ of respondents felt no need for revision of NEQIP. Over 80\% of respondents wanted simplified NEQIP criteria applicable in clinical practice, and approximately $2 / 3$ of respondents asked for streamlining the NEQIP criteria, clarifications of their definitions and performance targets, and scientific evidence supporting the NEQIP criteria.

Table 3. Characteristics of Sedatives for Endoscopy and Reprocessing of Endoscopes

\begin{tabular}{|c|c|}
\hline Characteristic & Value \\
\hline \multicolumn{2}{|l|}{ Sedative endoscopy* } \\
\hline \multicolumn{2}{|l|}{ Sedative agents } \\
\hline Benzodiazepine alone & $152(30.1)$ \\
\hline Propofol alone & $37(7.3)$ \\
\hline Combination of benzodiazepine and propofol & $294(58.2)$ \\
\hline Combination of etomidate and other agents & $17(3.4)$ \\
\hline Other agents & $5(1.0)$ \\
\hline Informed consent for sedative endoscopy & $481(95.2)$ \\
\hline Patient monitoring during sedative endoscopy & $486(96.2)$ \\
\hline \multicolumn{2}{|l|}{ Endoscopic reprocessing $^{\dagger}$} \\
\hline \multicolumn{2}{|l|}{ Disinfectants used } \\
\hline Glutaraldehyde & $111(27.5)$ \\
\hline Ortho-phthalaldehyde & 99 (24.5) \\
\hline Peracetic acid & $53(13.1)$ \\
\hline Superoxidized water & $28(6.9)$ \\
\hline Other agents or did not know main ingredient & $113(28.0)$ \\
\hline Sterilization of accessories that pass the mucosal barrier & $334(82.7)$ \\
\hline High-level disinfection of accessories attached to the endoscope & $343(84.9)$ \\
\hline Continuous medical education for endoscopic reprocessing & $340(84.2)$ \\
\hline
\end{tabular}

Data are presented as number $(\%)$.

${ }^{*}$ Two respondents were excluded from the analysis because they did not administer any sedatives for endoscopy; ${ }^{\dagger}$ One hundred three respondents were excluded from analysis because their survey responses were incomplete. 
Table 4. Opinions on the Revision of the National Endoscopy Quality Improvement Program

\begin{tabular}{|c|c|}
\hline Opinion for revision of NEQIP & Value $^{*}$ \\
\hline \multicolumn{2}{|l|}{ Familiarity with NEQIP } \\
\hline 1 (very familiar) & $57(15.4)$ \\
\hline 2 & $81(21.9)$ \\
\hline 3 & $130(35.1)$ \\
\hline 4 & $61(16.5)$ \\
\hline 5 (very unfamiliar) & $41(11.1)$ \\
\hline \multicolumn{2}{|l|}{ Reason for gap between NEQIP and clinical practice } \\
\hline Physician is not familiar with NEQIP & $120(32.4)$ \\
\hline Physician does not agree with NEQIP & $21(5.7)$ \\
\hline Physician cannot follow NEQIP in real practice & $161(43.5)$ \\
\hline Physician is not motivated due to insufficient reimbursement & $68(18.4)$ \\
\hline \multicolumn{2}{|l|}{ Opinion for current NEQIP } \\
\hline NEQIP helps improve quality of endoscopy & $211(57.0)$ \\
\hline NEQIP is supported by evidence-based studies & $173(46.8)$ \\
\hline NEQIP increases early diagnosis of gastric cancer or colorectal cancer & $223(60.3)$ \\
\hline NEQIP provides benefits for many of my patients & $255(68.9)$ \\
\hline NEQIP does not apply to many of my patients & $142(38.4)$ \\
\hline NEQIP is likely to be used in physician discipline & $247(66.8)$ \\
\hline NEQIP is likely to decrease the physician's reimbursement & $215(58.1)$ \\
\hline \multicolumn{2}{|l|}{ Opinion for the revision of the NEQIP } \\
\hline To clarify the definition and performance target of NEQIP & $252(68.1)$ \\
\hline To support scientific evidence of NEQIP & $247(66.8)$ \\
\hline To apply NEQIP easily in clinical practice & $299(80.8)$ \\
\hline To simplify NEQIP criteria & $256(69.2)$ \\
\hline
\end{tabular}

Data are presented as number (\%).

NEQIP, National Endoscopy Quality Improvement Program.

*One hundred thirty-seven respondents were excluded from the analysis because they provided incomplete responses to survey items.

\section{DISCUSSION}

This study presents the current status of the nationwide screening endoscopy, endoscopic capacity, sedation and reprocessing, as well as opinion for NEQIP in Korea. Of all physicians who responded to our survey, gastroenterologists performed most of the nationwide screening endoscopy in NCSP, and most of them had gastroenterology fellow training for more than 1 year with a sufficient cumulative endoscopy volume. Under current capacity of NCSP, typical waiting time for screening endoscopies in NCSP was less than 4 weeks in over 90\% of the endoscopy units, which was very short compared with those of other countries. ${ }^{12-14}$ In a survey of an Asian tertiary care hospital, ${ }^{12}$ $62 \%$ of patients had to wait for more than 4 weeks for their appointment, and a long waiting time was the main cause of patient dissatisfaction. In clinical practice guidelines established in Spain, the waiting time from positive FOBTs to colonoscopy was recommended to be less than 6 weeks. ${ }^{13}$ Furthermore, a Canadian consensus recommended screening colonoscopy should be performed within 6 months. ${ }^{14}$ The shorter waiting time for screening endoscopies in NCSP is likely due to the high number of available endoscopists and endoscopy units in Korea.

In the present survey, obstacles and strategies to accommodate an increased endoscopic demand in NCSP were questioned. The major obstacle was insufficient endoscopy reimbursement. As a result, most physicians were not likely to plan to perform more screening endoscopies in NCSP. For example, the medical cost of a screening EGD in NCSP was \$34.89 in 2008, ${ }^{15}$ which was approximately $1 / 20$ of the medical cost in the United States (\$685 for EGD alone). ${ }^{16}$ Our survey result may suggest that insufficient reimbursement could be a barrier to accommodate the increased endoscopic demand in the future. Furthermore, insufficient reimbursement may lead to a low quality of endoscopy. The next response to accommodate an increased endoscopic demand was that physicians would increase working hours for endoscopy, which was a similar result from the U.S. survey. ${ }^{17}$ Therefore, further studies optimizing reimbursement for screening endoscopy in NCSP are warranted in Korea. 
For sedation, benzodiazepine was the most commonly used form of sedation, either alone or in combination with other drugs, and propofol alone was used in only 7.3\% of patients. In a nationwide survey from Spain, ${ }^{18}$ benzodiazepine was used in 30\% of patients for EGD and colonoscopy, respectively, and propofol was used in 70\% of EGDs and in 80\% of colonoscopies, respectively. In a recent nationwide survey from Korea, ${ }^{19}$ benzodiazepine was used in combination with propofol in 25.3\% of EGDs and in 33.8\% of colonoscopies, and propofol alone was used in $29.0 \%$ of EGDs and $6.0 \%$ of colonoscopies, respectively. It seems likely that higher participation of endoscopists from primary clinic in that study ${ }^{19}$ may be a possible explanation for higher preference for propofol use compared with our data. The widespread use of propofol for gastrointestinal endoscopy has also been documented in several European countries. ${ }^{20,21}$ As the characteristics of propofol include a rapid onset of action with a short-term effect, a shorter recovery time, and greater patient and endoscopist satisfaction, ${ }^{22}$ propofol use may be preferred by endoscopists practicing in primary clinic setting. ${ }^{19}$ Our survey findings suggested suboptimal performance of endoscopy reprocessing, as $28 \%$ of respondents used unapproved disinfectants or did not know main ingredient of their disinfectants as well as $15 \%$ to $17 \%$ of respondents did not follow reprocessing protocols. ${ }^{23}$ A Chinese survey from 122 endoscopy units and an Italian survey from 278 endoscopy units also showed significant room for improvement in the practice of endoscopic reprocessing..$^{24,25}$ Our survey findings suggest that there are substantial gaps between clinical practice and reprocessing guideline recommendations, and highlight the need for improving performance of reprocessing protocols.

Physician awareness, familiarity, and agreement were potential barriers to NEQIP adherence. ${ }^{26}$ Our survey findings showed relatively optimal agreement of NEQIP as only 5.7\% of respondents do not agree with NEQIP, however, its familiarity was suboptimal as only $37.3 \%$ of respondents were familiar with NEQIP criteria. Therefore, efforts to improve familiarity with NEQIP may be necessary. Low familiarity with NEQIP may partially be explained by that physicians concerned that NEQIP would likely be used in physician discipline and thus decrease physician reimbursement.

Our survey results need to be interpreted in the context of the potential limitations. The generalizability of this study may be limited as respondents' response tendencies may differ that from nonrespondents or variable practice settings. In addition, as we limited the study population to endoscopists who performed screening endoscopy in NCSP, our results may therefore not be generalizable to other endoscopists who do not perform endoscopy in NCSP. Like most survey study, this survey also totally relied on the knowledge and the answer of the respondents. Even though, our study has several strengths. First, this is the first study on the current status of the NCSP, endoscopic capacity, sedation and reprocessing as well as endoscopists' opinions for NEQIP in Korea. Second, the questionnaire development was performed by discussion with academic and community gastroenterologists, and reviewed by members of the Ethics and Quality Improvement Committee of KSGE. For this survey, we used a number of evidence-based strategies to maximize responses, including short survey length, multiple contacts, and incentives. Finally, a large number of gastroenterologists responded to our survey from various institutional levels. Therefore, our survey results may represent the universal opinion of all gastroenterologists in Korea.

In conclusion, NEQIP is still suboptimal in Korea. Considering suboptimal performance of endoscopy reprocessing and low awareness and agreement of NEQIP, the implementation of quality in endoscopy reprocessing and a better understanding of NEQIP should be underlined in Korea.

\section{CONFLICTS OF INTEREST}

No potential conflict of interest relevant to this article was reported.

\section{ACKNOWLEDGEMENTS}

This study was supported by National Cancer Center grant (1560460-1).

\section{REFERENCES}

1. Shin A, Kim J, Park S. Gastric cancer epidemiology in Korea. J Gastric Cancer 2011;11:135-140.

2. Choi KS, Jun JK, Park EC, et al. Performance of different gastric cancer screening methods in Korea: a population-based study. PLoS One 2012;7:e50041.

3. Shin A, Choi KS, Jun JK, et al. Validity of fecal occult blood test in the national cancer screening program, Korea. PLoS One 2013;8: e79292.

4. Yoo KY. Cancer control activities in the Republic of Korea. Jpn J Clin Oncol 2008;38:327-333.

5. National Cancer Center, Education for National Cancer Screening Program [Internet]. Goyang: National Cancer Center; c2015 [cited 2015 Dec 26]. Available from: http://education.ncc.re.kr/main.do.

6. Lee S, Jun JK, Suh M, et al. Gastric cancer screening uptake trends in Korea: results for the National Cancer Screening Program from 2002 to 2011: a prospective cross-sectional study. Medicine (Baltimore) 2015;94:e533.

7. Cha JM. Quality improvement of gastrointestinal endoscopy in Korea: past, present, and future. Korean J Gastroenterol 2014;64: 320-332.

8. Cha JM, Han DS, Lee HL, et al. Endoscopist specialty is associated with high-quality endoscopy in Korea. Yonsei Med J 2012;53:310317.

9. Park WG, Shaheen NJ, Cohen J, et al. Quality indicators for EGD. 
Am J Gastroenterol 2015;110:60-71.

10. Rex DK, Schoenfeld PS, Cohen J, et al. Quality indicators for colonoscopy. Am J Gastroenterol 2015;110:72-90.

11. Rembacken B, Hassan C, Riemann JF, et al. Quality in screening colonoscopy: position statement of the European Society of Gastrointestinal Endoscopy (ESGE). Endoscopy 2012;44:957-968.

12. Azmi N, Chan WK, Goh KL. Evaluation of patient satisfaction of an outpatient gastroscopy service in an Asian tertiary care hospital. BMC Gastroenterol 2012;12:96.

13. Jover R, Herráiz M, Alarcón 0 , et al. Clinical practice guidelines: quality of colonoscopy in colorectal cancer screening. Endoscopy 2012;44:444-451.

14. Paterson WG, Depew WT, Paré P, et al. Canadian consensus on medically acceptable wait times for digestive health care. Can J Gastroenterol 2006;20:411-423.

15. Lee HY, Park EC, Jun JK, Choi KS, Hahm MI. Comparing upper gastrointestinal X-ray and endoscopy for gastric cancer diagnosis in Korea. World J Gastroenterol 2010;16:245-250.

16. Peery AF, Dellon ES, Lund J, et al. Burden of gastrointestinal disease in the United States: 2012 update. Gastroenterology 2012;143:1179-1187.e3.

17. Seeff LC, Richards TB, Shapiro JA, et al. How many endoscopies are performed for colorectal cancer screening? Results from CDC's survey of endoscopic capacity. Gastroenterology 2004;127:16701677.

18. Lucendo AJ, González-Huix F, Tenias JM, et al. Gastrointestinal endoscopy sedation and monitoring practices in Spain: a nationwide survey in the year 2014. Endoscopy 2015;47:383-390.
19. Lee CK, Dong SH, Kim ES, et al. Room for quality improvement in endoscopist-directed sedation: results from the first nationwide survey in Korea. Gut Liver 2016;10:83-94.

20. Riphaus A, Geist F, Wehrmann T. Endoscopic sedation and monitoring practice in Germany: re-evaluation from the first nationwide survey 3 years after the implementation of an evidence and consent based national guideline. Z Gastroenterol 2013;51:10821088.

21. Repici A, Pagano N, Hassan C, et al. Balanced propofol sedation administered by nonanesthesiologists: the first Italian experience. World J Gastroenterol 2011;17:3818-3823.

22. McQuaid KR, Laine L. A systematic review and meta-analysis of randomized, controlled trials of moderate sedation for routine endoscopic procedures. Gastrointest Endosc 2008;67:910-923.

23. Park S, Jang JY, Koo JS, et al. A review of current disinfectants for gastrointestinal endoscopic reprocessing. Clin Endosc 2013;46: 337-341.

24. Zhang X, Kong J, Tang P, et al. Current status of cleaning and disinfection for gastrointestinal endoscopy in China: a survey of 122 endoscopy units. Dig Liver Dis 2011;43:305-308.

25. Radaelli F, Meucci G, Minoli G; Italian Association of Hospital Gastroenterologists (AIGO). Colonoscopy practice in Italy: a prospective survey on behalf of the Italian Association of Hospital Gastroenterologists. Dig Liver Dis 2008;40:897-904.

26. Ellett ML. A literature review of the safety and efficacy of using propofol for sedation in endoscopy. Gastroenterol Nurs 2010;33: 111-117. 